\title{
CLOSED-FORM ERROR ESTIMATES FOR THE NUMERICAL SOLUTION OF FREDHOLM INTEGRAL EQUATIONS OF THE SECOND KIND
}

\author{
CHRISTIAN M. GROH AND MARK A. KELMANSON \\ Communicated by Kendall Atkinson
}

\begin{abstract}
Closed-form algebraic formulae are derived for the local error incurred in the numerical solution of integral equations by iterated collocation methods, the analysis being illustrated by application to Fredholm integral equations of the second kind. The novel error analysis uses an asymptotic approach, in the small parameter of the numerical mesh size, applied to a finite-rank degenerate-kernel orthogonalpolynomial approximation of the exact kernel. It is proved that, under suitable conditions, the discrepancy between our theoretically predicted error and the actual numerical error tends to zero at the rate $\left\|\mathcal{K}-\mathcal{K}_{M}\right\|$, where $M$ is the rank of the degenerate-kernel approximation. A leading-order error analysis is validated on three increasingly accurate projection methods applied to both smooth and sharply peaked kernels, our error predictions being demonstrated to be exponentially convergent to experimentally obtained global numerical errors. The new method is demonstrated to be cost-effective relative to standard extrapolation.
\end{abstract}

1. Introduction. This paper is concerned with obtaining explicit, closed-form, algebraic expressions for the error incurred in the numerical solution of the Fredholm integral equation of the second kind,

$$
\phi(x)=f(x)+\lambda \int_{a}^{b} K(x, s) \phi(s) d s
$$

for a variety of approximating projection methods. Although degeneratekernel (and orthogonal-polynomial) techniques do indeed constitute an

Keywords and phrases. Fredholm integral equations; error analysis; degenerate kernels; orthogonal polynomials

Received by the editors on July 7, 2006, and in revised form on February 26, 2007.

DOI:10.1216/JIE-2008-20-4-481 Copyright (C)2008 Rocky Mountain Mathematics Consortium 
element of our overall perturbation analysis, it is to be stressed at the outset that we do not specifically analyze degenerate-kernel methods, in which there is a substantial and well-established literature (e.g., Atkinson (1997, Ch.2), Baker (1997, §4.7), Dellwo (1995), Hackbush (1995, $\S 4.2 .1)$, Heinrich (1985), Kress (1989, Ch.11), Sloan et al. (1975), Sloan (1976a) and Sloan (1976b)). Rather, the present work is a novel error analysis of practical projection methods commonly employed in determining approximate solutions of (1), in which $f:[a, b] \rightarrow \mathbb{R}$ and the kernel $K:[a, b] \times[a, b] \rightarrow \mathbb{R}$ are prescribed functions, and $\lambda, a, b \in \mathbb{R}$ are constants. We rewrite (1) in symbolic form as

$$
\phi=f+\lambda \mathcal{K} \phi,
$$

in which $\phi, f \in C[a, b]$, the Banach space with supremum norm $\|\bullet\|$ on which $\mathcal{K}$ is a compact linear operator; it is assumed here, and throughout the rest of the paper, that $\lambda$ is not a characteristic value of (2). We correspondingly denote by $\phi^{(n)}$ the solution of the iterated collocation approximation of (2) associated with the $n^{\text {th }}$-degree piecewise-polynomial interpolatory projection operator $\mathcal{P}_{h}^{(n)}$ on a mesh of size $h$, i.e.

$$
\phi^{(n)}=f+\lambda \mathcal{K} \mathcal{P}_{h}^{(n)} \phi^{(n)} .
$$

In this paper we consider interpolation with $n=0,1$ and 2 , using mid-, end- and end-point rules respectively. If the kernel $K(x, s)$ can be approximated by the finite-rank degenerate kernel

$$
K_{M}(x, s)=\sum_{m=1}^{M} \zeta_{m}(x) \sigma_{m}(s) \quad(M \geq 1),
$$

then we denote by $\phi_{M}$ the exact solution of the corresponding degeneratekernel approximation of (2),

$$
\phi_{M}=f+\lambda \mathcal{K}_{M} \phi_{M},
$$

and by $\phi_{M}^{(n)}$ the solution of the approximation of (5) associated with the projection operator $\mathcal{P}_{h}^{(n)}$,

$$
\phi_{M}^{(n)}=f+\lambda \mathcal{K}_{M} \mathcal{P}_{h}^{(n)} \phi_{M}^{(n)} .
$$


It is to be understood throughout that $\phi^{(n)}$ and $\phi_{M}^{(n)}$ are also dependent upon $h$ despite the absence of such indication in the notation.

In the present paper, we estimate the actual numerical error $\mathcal{E}^{(n)} \equiv$ $\phi-\phi^{(n)}$ in the iterated projection method (3) via the intermediate evaluation of a theoretical error $\mathcal{E}_{M}^{(n)} \equiv \phi_{M}-\phi_{M}^{(n)}$ for which explicit, closed-form formulae are determined. This explains the apparently unusual step of considering the projected equation (6), of the degeneratekernel equation (5), whose projection we never actually undertake in a computational sense. The idea is summarized in the commutative diagram (7).

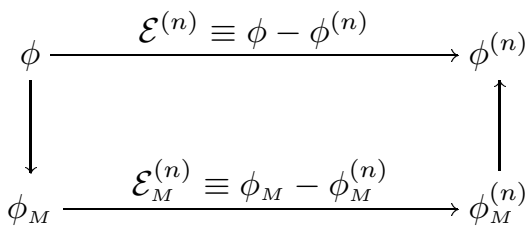

The actual numerical error $\mathcal{E}^{(n)}$ is approximated by the theoretical error $\mathcal{E}_{M}^{(n)}$ using the path from $\phi$ to $\phi^{(n)}$ via $\phi_{M}$ and (the virtual) $\phi_{M}^{(n)}$. As such, we prove that $\mathcal{E}_{M}^{(n)}$ converges to $\mathcal{E}^{(n)}$ as $M \rightarrow \infty$, and we demonstrate that in practical calculations, this convergence can be achieved economically, i.e. for low values of $M$. Note that here $\mathcal{E}_{M}^{(n)}(x)$ is determined as a function of $x$ throughout the interval $[a, b]$. Numerical estimates of $\left\|\mathcal{E}^{(n)}\right\|$ are difficult to evaluate in applications (Kress, 1989, p.158), often requiring convergence estimates obtained from mesh refinement and extrapolation. Even if direct error estimates can be obtained, there may be a disparity in magnitude between the true and predicted errors (Atkinson, 1997, p.33). The estimates herein are novel because they are obtained as leading-order terms in an explicit perturbation analysis, rather than via more conservative estimates of condition numbers (Linz, 1991; Whitley, 1986) based upon operator representations of (2) and (3). The estimates are furthermore practical and useful because of the above-mentioned convergence for low values of $M$ and the known difficulty in determining accurate estimates of $\mathcal{E}^{(n)}=\phi-\phi^{(n)}$.

The rest of the paper is structured as follows. The basic elements of our perturbation theory are introduced in $\S 2$ by considering the simplest possible degeneracy, with $M=1$. This error analysis is 
extended in $\S 3$ to the case $M>1$, with the aim of applying our analysis to non-degenerate kernels approximated by degenerate ones of adequately high rank. In $\S 4$ a formal error analysis is presented for non-degenerate kernels in order to provide a theoretical framework for our new method, the implementational details of which are summarized in $\S 5$. In $\S 6$, the accuracy of our new approach is demonstrated via application to two examples of integral equations incorporating smooth and sharply peaked kernels. The paper concludes in $\S 7$ with a discussion of the utility of the new method by performing cost comparisons with standard extrapolation techniques.

2. Error analysis for $\mathbf{M}=\mathbf{1}$. It is instructive to begin with the case of a trivially separable kernel $(M \equiv 1)$ in $(4)$, when we define

$$
\left(\mathcal{K}_{1} F\right)(x) \equiv \int_{a}^{b} \zeta_{1}(x) \sigma_{1}(s) F(s) d s \quad \text { and } \quad\langle F, G\rangle \equiv \int_{a}^{b} F(x) G(x) d x,
$$

where $F$ and $G$ are suitable functions $F, G: \mathbb{R} \rightarrow \mathbb{R}:$. It is straightforward to show that (5),

$$
\phi_{1}=f+\lambda \mathcal{K}_{1} \phi_{1},
$$

has solution

$$
\phi_{1}=f+\frac{\left\langle\sigma_{1}, f\right\rangle}{1-\left\langle\sigma_{1}, \xi_{1}\right\rangle} \xi_{1}
$$

where $\xi_{1}(x)=\lambda \zeta_{1}(x)$. Note that (9) is valid because $\lambda$ is not a characteristic value of (8), i.e.

$$
\lambda \neq \frac{1}{\left\langle\sigma_{1}, \zeta_{1}\right\rangle} .
$$

Similarly, (6),

$$
\phi_{1}^{(n)}=f+\lambda \mathcal{K}_{1} \mathcal{P}_{h}^{(n)} \phi_{1}^{(n)},
$$

has solution

$$
\phi_{1}^{(n)}=f+\frac{\left\langle\sigma_{1}, \mathcal{P}_{h}^{(n)} f\right\rangle}{1-\left\langle\sigma_{1}, \mathcal{P}_{h}^{(n)} \xi_{1}\right\rangle} \xi_{1},
$$


where the projection operator $\mathcal{P}_{h}^{(n)} \phi$, as yet unspecified, dictates the piecewise-polynomial interpolation (of degree $n$ ) at the finite set of node points $\left(x_{i}, \phi_{i}\right), i=1,2, \ldots, N+\frac{n}{2}(3-n)$ that lie on a regular mesh of size $h=(b-a) / N$, the node locations being mid-interval for $n=0$ and interval endpoints for $n=1$ and $n=2$. Now define

$$
\delta^{(n)}\left(\sigma_{1}, \bullet\right) \equiv\left\langle\sigma_{1}, \bullet\right\rangle-\left\langle\sigma_{1}, \mathcal{P}_{h}^{(n)} \bullet\right\rangle=\left\langle\sigma_{1},\left(\mathcal{I}-\mathcal{P}_{h}^{(n)}\right) \bullet\right\rangle .
$$

Via the Cauchy-Schwarz inequality and standard results on piecewise constant, linear and quadratic (respectively $n=0,1$ and 2) polynomial interpolation, (13) gives (cf. Atkinson (1997, (2.3.48) and (3.4.107)))

$$
\begin{aligned}
\left|\delta^{(n)}\left(\sigma_{1}, g\right)\right| & =\left|\left\langle\sigma_{1}, g-\mathcal{P}_{h}^{(n)} g\right\rangle\right| \leq\left\|\sigma_{1}\right\|\left\|g-\mathcal{P}_{h}^{(n)} g\right\| \\
& \leq \Omega^{(n)}\left\|\sigma_{1}\right\|\left\|\frac{d^{n+1} g}{d x^{n+1}}\right\| h^{n+1},
\end{aligned}
$$

in which $\Omega^{(n)}=\frac{1}{2}, \frac{1}{8}$ and $\frac{\sqrt{3}}{27}$ for $n=0,1$ and 2. Hence, via (9) and (12), the estimate (14) predicts

$$
\phi_{1}-\phi_{1}^{(n)}=\mathcal{O}\left(h^{n+1}\right)
$$

provided both $\sigma_{1}, g \in C^{n+1}[a, b]$. Direct calculation, however, yields

$$
\delta^{(n)}\left(\sigma_{1}, g\right)=\chi_{0}^{(n)}\left(\sigma_{1}, g\right) h^{\nu_{n}}+\mathcal{O}\left(h^{\nu_{n}+2}\right)
$$

where we find $\nu_{n}=n^{2}-n+2$ for $n=0,1,2$, and ${ }^{2}$

$$
\chi_{0}^{(n)}\left(\sigma_{1}, g\right)=\left\{\begin{array}{lll}
\frac{1}{24} \int_{a}^{b}\left(2 \sigma_{1}^{\prime} g^{\prime}+\sigma_{1} g^{\prime \prime}\right) d x, & n=0 & \left(\nu_{n}=2\right), \\
-\frac{1}{12} \int_{a}^{b} \sigma_{1} g^{\prime \prime} d x, & n=1 & \left(\nu_{n}=2\right), \\
-\frac{1}{180} \int_{a}^{b}\left(4 \sigma_{1}^{\prime} g^{\prime \prime \prime}+\sigma_{1} g^{(i v)}\right) d x, & n=2 & \left(\nu_{n}=4\right) .
\end{array}\right.
$$

\footnotetext{
2 The integral forms of the coefficients in (17) are a result of midpoint-rule integration over $[a, b]$ of all local truncation errors. As such, the $\Xi_{0}^{(n)}$ themselves have truncation errors that are series in $h^{2}$. Hence the asymptotic approximation (16) is consistent as presented.
} 
Hence when $\sigma_{1}$ is a polynomial, (16) holds provided $g \in C^{\nu_{n}}[a, b]$. Under these conditions, and for sufficiently small $h, \delta^{(n)}\left(\sigma_{1},{ }^{\bullet}\right)$ as defined in (13) can be used as an expansion parameter. Accordingly, from (9), (12) and (16), we obtain the explicit leading-order error estimate

(18) $\phi_{1}-\phi_{1}^{(n)}=\alpha \xi_{1}\left(\delta^{(n)}\left(\sigma_{1}, f\right)+\alpha\left\langle\sigma_{1}, f\right\rangle \delta^{(n)}\left(\sigma_{1}, \xi_{1}\right)\right)+\mathcal{O}\left(h^{\nu_{n}+2}\right)$,

where $\alpha=1 /\left(1-\left\langle\sigma_{1}, \xi_{1}\right\rangle\right)$ and both $\delta^{(n)}\left(\sigma_{1}, f\right)$ and $\delta^{(n)}\left(\sigma_{1}, \xi_{1}\right)$ are calculated via (13). Note that the integrated coefficients implicit in (18) yield a tighter error bound than (15) when $n=0$ or 2 . Although the truncated terms of order $\mathcal{O}\left(h^{\nu_{n}+2}\right)$ and higher in (16) may be obtained by similar evaluation of integral coefficients $\chi_{j}^{(n)}\left(\sigma_{1}, g\right)$ for $j \geq 1$, these rapidly become algebraically expensive with increasing $j$ and $n$. More importantly, as we demonstrate below, adherence to the leading-order expression (16) admits a linearized theory that is both asymptotically consistent to order $\mathcal{O}\left(h^{\nu_{n}}\right)$ and, as evidenced by the results in $\S 6$, yields error discrepancies that are exponentially convergent with $M$ until the truncation plateau, due to the omission of terms of order $\mathcal{O}\left(h^{\nu_{n}+2}\right)$ in (18), is reached. The analysis for $M=1$ complete, we now progress to the case $M>1$ in order to analyze the approximation of non-degenerate kernels.

3. Error analysis for $\mathbf{M}>\mathbf{1}$. Equation (5) may be written in the expanded form

$$
\phi_{M}(x)=f(x)+\sum_{m=1}^{M} \xi_{m}(x) \int_{a}^{b} \sigma_{m}(s) \phi_{M}(s) d s,
$$

its approximation (6) being

$$
\phi_{M}^{(n)}(x)=f(x)+\sum_{m=1}^{M} \xi_{m}(x) \int_{a}^{b} \sigma_{m}(s) \mathcal{P}_{h}^{(n)} \phi_{M}^{(n)}(s) d s .
$$

Explicit solutions of (19) and (20) are given by

(21) $\phi_{M}(x)=f(x)+\sum_{m=1}^{M} c_{m} \xi_{m}(x)$ and $\phi_{M}^{(n)}(x)=f(x)+\sum_{m=1}^{M} c_{m}^{(n)} \xi_{m}(x)$ 
respectively, for some constants $c_{m}, c_{m}^{(n)} \in \mathbb{R}, m=1, \ldots, M$, so that (21) gives the error in $\phi_{M}^{(n)}(x)$ explicitly as

$$
\mathcal{E}_{M}^{(n)}=\phi_{M}(x)-\phi_{M}^{(n)}(x)=\sum_{m=1}^{M} \delta c_{m}^{(n)} \xi_{m}(x),
$$

where $\delta c_{m}^{(n)} \equiv c_{m}-c_{m}^{(n)}, m=1, \ldots, M$. The coefficients $c_{m}$ and $c_{m}^{(n)}$ are (e.g., Hildebrand $(1952, \S 4.6))$ respectively solutions of the linear simultaneous systems

$$
\begin{aligned}
\sum_{j=1}^{M}\left(\delta_{i j}-\left\langle\sigma_{i}, \xi_{j}\right\rangle\right) c_{j} & =\left\langle\sigma_{i}, f\right\rangle, \\
\sum_{j=1}^{M}\left(\delta_{i j}-\left\langle\sigma_{i}, \mathcal{P}_{h}^{(n)} \xi_{j}\right\rangle\right) c_{j}^{(n)} & =\left\langle\sigma_{i}, \mathcal{P}_{h}^{(n)} f\right\rangle,
\end{aligned}
$$

where $i=1 \ldots M$ and $\delta_{i j}$ is the Kronecker delta. Using (13), (24) may be rewritten as

$$
\sum_{j=1}^{M}\left(\delta_{i j}-\left\langle\sigma_{i}, \xi_{j}\right\rangle+\delta^{(n)}\left(\sigma_{i}, \xi_{j}\right)\right) c_{j}^{(n)}=\left\langle\sigma_{i}, f\right\rangle-\delta^{(n)}\left(\sigma_{i}, f\right) .
$$

Rewriting (23) and (25) in an obvious notation, we have

$$
\begin{aligned}
(\mathbf{I}-\mathbf{X}) \mathbf{C} & =\mathbf{F} \\
(\mathbf{I}-\mathbf{X}+\delta \mathbf{X}) \mathbf{C}^{(n)} & =(\mathbf{I}-\mathbf{X}+\delta \mathbf{X})\left(\mathbf{C}-\delta \mathbf{C}^{(n)}\right)=\mathbf{F}-\delta \mathbf{F}
\end{aligned}
$$

Subtracting equation (27) from (26) yields

$$
(\mathbf{I}-\mathbf{X}+\delta \mathbf{X}) \delta \mathbf{C}^{(n)}=\delta \mathbf{F}+\delta \mathbf{X} \mathbf{C},
$$

and hence

$$
\delta \mathbf{C}^{(n)}=(\mathbf{I}-\mathbf{X}+\delta \mathbf{X})^{-1}(\delta \mathbf{F}+\delta \mathbf{X} \mathbf{C})
$$


in which the parameter $\lambda$ in (2) is incorporated in the matrices $\mathbf{X}$ and $\delta \mathbf{X}$ through the functions $\xi_{j}(x)=\lambda \zeta_{j}(x), j=1, \ldots, M$. By hypothesis, $\lambda$ is not a characteristic value of (5), equivalently (19), hence the matrix $\mathbf{I}-\mathbf{X}$ in (26) is invertible, and so (Golub and Van Loan, 1989, Theorem 2.3.4) $(\mathbf{I}-\mathbf{X}+\delta \mathbf{X})$ is also invertible provided

$$
\kappa \equiv\left\|(\mathbf{I}-\mathbf{X})^{-\mathbf{1}} \delta \mathbf{X}\right\|<1 .
$$

By construction, the elements of the $M \times M$ matrix $\delta \mathbf{X}$ are of order $\mathcal{O}\left(h^{\nu_{n}}\right)$. If, in the absence of any other information, we make the naïve assumption that the elements of the $M \times M$ matrix $(\mathbf{I}-\mathbf{X})^{-1}$ are of order $\mathcal{O}(1),(30)$ holds provided that $\mathcal{O}\left(M^{2} h^{\nu_{n}}\right)<1$, equivalently

$$
M<\mathcal{O}\left(N^{\nu_{n} / 2}\right) .
$$

However, as the results and discussion in $\S 6$ will reveal, although it is indeed the case that $\left|[\delta \mathbf{X}]_{i j}\right|$ is of order $\mathcal{O}\left(h^{\nu_{n}}\right)$ for $1 \leq i, j \leq M$, it is actually the case that $\left|\left[(\mathbf{I}-\mathbf{X})^{-1}\right]_{i j}\right|$ is of order $\mathcal{O}(1)$ when $i=j=1$ and decreases exponentially with increasing $i$ and $j$, so that $(\mathbf{I}-\mathbf{X})^{-1}$ is in practice a perturbed identity matrix. This has two implications. First, (30) then yields $\kappa \approx\|\delta \mathbf{X}\| \approx M h^{\nu_{n}}$, in which case the naïve bound (31) is relaxed considerably to

$$
M<\mathcal{O}\left(N^{\nu_{n}}\right),
$$

which will in practice always be met since the aim of the method is to produce useful error estimates for low values of $M$. Second, it results in the elements of $\delta \mathbf{C}^{(n)}$ being of order $\mathcal{O}\left(h^{\nu_{n}}\right)$, in which event it is instructive to examine the effect of linearizing (28) by omitting the quadratic term. By so doing, $\delta \mathbf{C}^{(n)}$ may be determined more economically in terms of the single inverse $(\mathbf{I}-\mathbf{X})^{-1}$ in (29). To this end, let $\delta \mathbf{C}_{L}^{(n)}$ satisfy the linearized version of (29),

$$
\delta \mathbf{C}_{L}^{(n)}=(\mathbf{I}-\mathbf{X})^{-1}(\delta \mathbf{F}+\delta \mathbf{X} \mathbf{C}) .
$$

Note that

$$
\begin{aligned}
(\mathbf{I}-\mathbf{X}+\delta \mathbf{X})^{-1} & =\left\{(\mathbf{I}-\mathbf{X})\left[\mathbf{I}+(\mathbf{I}-\mathbf{X})^{-\mathbf{1}} \delta \mathbf{X}\right]\right\}^{-1} \\
& =\left[\mathbf{I}+(\mathbf{I}-\mathbf{X})^{-\mathbf{1}} \delta \mathbf{X}\right]^{-\mathbf{1}}(\mathbf{I}-\mathbf{X})^{-1}
\end{aligned}
$$


Since (31) guarantees that (30) will be met, we have (Golub and Van Loan, 1989, Lemma 2.3.3)

$$
\left[\mathbf{I}+(\mathbf{I}-\mathbf{X})^{-\mathbf{1}} \delta \mathbf{X}\right]^{-1}=\sum_{k=0}^{\infty}(-1)^{k}\left[(\mathbf{I}-\mathbf{X})^{-\mathbf{1}} \delta \mathbf{X}\right]^{k}
$$

Hence when (31) is satisfied, (29) yields

$$
\delta \mathbf{C}^{(n)}=\left\{\sum_{k=0}^{\infty}(-1)^{k}[\mathbf{Y} \delta \mathbf{X}]^{k}\right\} \mathbf{Y}(\delta \mathbf{F}+\delta \mathbf{X} \mathbf{C}),
$$

in which the existence of $\mathbf{Y} \equiv(\mathbf{I}-\mathbf{X})^{-\mathbf{1}}$ is ensured by hypothesis. Based upon (16), substitution of

$$
\begin{aligned}
\delta \mathbf{X} & =h^{\nu_{n}} \delta \mathbf{X}_{\mathbf{0}}+h^{\nu_{n}+2} \delta \mathbf{X}_{\mathbf{1}}+\mathcal{O}\left(h^{\nu_{n}+4}\right) \\
\delta \mathbf{F} & =h^{\nu_{n}} \delta \mathbf{F}_{\mathbf{0}}+h^{\nu_{n}+2} \delta \mathbf{F}_{\mathbf{1}}+\mathcal{O}\left(h^{\nu_{n}+4}\right)
\end{aligned} \quad \text { and }
$$

into (34) gives, when $n=0$ and $n=1$,

$$
\begin{aligned}
\delta \mathbf{C}^{(n)}= & h^{2} \mathbf{Y}\left(\delta \mathbf{F}_{\mathbf{0}}+\delta \mathbf{X}_{\mathbf{0}} \mathbf{C}\right) \\
& +h^{4}\left[\mathbf{Y}\left(\delta \mathbf{F}_{\mathbf{1}}+\delta \mathbf{X}_{\mathbf{1}} \mathbf{C}\right)-\mathbf{Y} \delta \mathbf{X}_{\mathbf{0}} \mathbf{Y}\left(\delta \mathbf{F}_{\mathbf{0}}+\delta \mathbf{X}_{\mathbf{0}} \mathbf{C}\right)\right]+\mathcal{O}\left(h^{6}\right) \\
= & \mathbf{Y}(\delta \mathbf{F}+\delta \mathbf{X} \mathbf{C})+\mathcal{O}\left(h^{4}\right)=\delta \mathbf{C}_{L}^{(n)}+\mathcal{O}\left(h^{2 \nu_{n}}\right)
\end{aligned}
$$

Similarly, when $n=2$ we have

$$
\begin{aligned}
\delta \mathbf{C}^{(n)} & =h^{4} \mathbf{Y}\left(\delta \mathbf{F}_{\mathbf{0}}+\delta \mathbf{X}_{\mathbf{0}} \mathbf{C}\right)+h^{6} \mathbf{Y}\left(\delta \mathbf{F}_{\mathbf{1}}+\delta \mathbf{X}_{\mathbf{1}} \mathbf{C}\right)+\mathcal{O}\left(h^{8}\right) \\
& =\mathbf{Y}(\delta \mathbf{F}+\delta \mathbf{X} \mathbf{C})+\mathcal{O}\left(h^{8}\right)=\delta \mathbf{C}_{L}^{(n)}+\mathcal{O}\left(h^{2 \nu_{n}}\right)
\end{aligned}
$$

Hence for all $n=0,1,2$, the linearized $\delta \mathbf{C}_{L}^{(n)}$ has the correct leadingorder behavior of $\delta \mathbf{C}^{(n)}$ and a first-order relative error of order $\mathcal{O}\left(h^{\nu_{n}}\right)$, at which order the coefficient is itself of order $\mathcal{O}(1)$ by virtue of the constraint (31). Numerical corroboration of this assertion is given in $\S 6$, to which further discussion of this matter is deferred.

Accordingly, in $\S \S 5$ and 6 , we implement the full, non-linear (29) that requires no a priori assumptions about the magnitude of $\left\|\delta \mathbf{C}^{(n)}\right\|$. In 
(29), $\delta \mathbf{X}$ and $\delta \mathbf{F}$ are, by (13) and (25), given by $(\delta \mathbf{X})_{i j}=\delta^{(n)}\left(\sigma_{i}, \xi_{j}\right)$ and $(\delta \mathbf{F})_{i}=\delta^{(n)}\left(\sigma_{i}, f\right)$, each of which may be determined using (13) and (17). With $\delta \mathbf{C}^{(n)}$ thus determined via (29), (22) in the form

$$
\mathcal{E}_{M}^{(n)} \approx \sum_{i=1}^{M} \delta \mathbf{C}_{i}^{(n)} \xi_{i}
$$

provides the explicit leading-order behavior of the error $\mathcal{E}_{M}^{(n)}$ in the degenerate-kernel approximation.

4. Error analysis for non-degenerate kernels. In order to establish the theoretical framework in which our method works, we rewrite (2) to (6) as

$$
\begin{aligned}
\mathcal{A} \phi & \equiv(\mathcal{I}-\lambda \mathcal{K}) \phi=f \\
\mathcal{B} \phi_{M} & \equiv\left(\mathcal{I}-\lambda \mathcal{K}_{M}\right) \phi_{M}=f \\
\mathcal{C} \phi^{(n)} & \equiv\left(\mathcal{I}-\lambda \mathcal{K} \mathcal{P}_{h}^{(n)}\right) \phi^{(n)}=f \\
\mathcal{D} \phi_{M}^{(n)} & \equiv\left(\mathcal{I}-\lambda \mathcal{K}_{M} \mathcal{P}_{h}^{(n)}\right) \phi_{M}^{(n)}=f .
\end{aligned}
$$

All unattributed external references within the remainder of this section are to Atkinson (1997). It is assumed that $0<\|f\|<\infty, \mathcal{K}$ is compact on $C[a, b]$ with the supremum norm, and $\mathcal{A}$ has a bounded inverse. By Theorem 2.1.1 $\mathcal{B}$ is invertible because, by construction,

$$
\left\|\mathcal{K}-\mathcal{K}_{M}\right\| \equiv \max _{x \in[a, b]} \int_{a}^{b}\left|K(x, s)-K_{M}(x, s)\right| d s
$$

satisfies

$$
\lim _{M \rightarrow \infty}\left\|\mathcal{K}-\mathcal{K}_{M}\right\|=0
$$

Equations (40) and (41) give

$$
\mathcal{D}=\mathcal{C}+\lambda\left(\mathcal{K}-\mathcal{K}_{M}\right) \mathcal{P}_{h}^{(n)}=\mathcal{C}\left(\mathcal{I}+\lambda \mathcal{C}^{-1}\left(\mathcal{K}-\mathcal{K}_{M}\right) \mathcal{P}_{h}^{(n)}\right)
$$


and so $\left\|\mathcal{D}^{-1}\right\|$ exists and is bounded if, via the geometric series theorem (Baker, 1977, p.51),

$$
\left\|\mathcal{K}-\mathcal{K}_{M}\right\| \leq \frac{1}{|\lambda|\left\|\mathcal{C}^{-1}\right\|\left\|\mathcal{P}_{h}^{(n)}\right\|} .
$$

By (2.1.11), this bound can be met for sufficiently large $M$ provided $\left\|\mathcal{C}^{-1}\right\|$ is bounded. For the piecewise-polynomial projections presently used, standard results on polynomial approximation give

$$
\left\|\mathcal{K}-\mathcal{P}_{h}^{(n)} \mathcal{K}\right\| \leq \Omega^{(n)} h^{n+1} \int_{a}^{b} \max _{x \in[a, b]}\left|\frac{\partial^{n+1}}{\partial x^{n+1}} K(x, s)\right| d s
$$

where $\Omega^{(n)}=\frac{1}{2}, \frac{1}{8}$ and $\frac{\sqrt{3}}{27}$ for $n=0,1$ and 2. Hence if $K(x, s)$ is $(n+1)$ times continuously differentiable with respect to $x$, we have ${ }^{3}$

$$
\lim _{h \rightarrow 0}\left\|\mathcal{K}-\mathcal{P}_{h}^{(n)} \mathcal{K}\right\|=0,
$$

and so condition (3.1.28) of Theorem 3.1.1 is met. Hence $(\mathcal{I}-$ $\left.\lambda \mathcal{P}_{h}^{(n)} \mathcal{K}\right)^{-1}$ exists and is uniformly bounded for sufficiently small $h$ so that, by lemma 3.4.1, $\left(\mathcal{I}-\lambda \mathcal{K} \mathcal{P}_{h}^{(n)}\right)^{-1}=\mathcal{C}^{-1}$ exists and is bounded for sufficiently small $h$. The error discrepancy, the difference between the true numerical error and predicted numerical error, is

$$
\begin{aligned}
\Delta \mathcal{E}_{M}^{(n)} & \equiv \mathcal{E}^{(n)}-\mathcal{E}_{M}^{(n)} \\
& =\left(\phi-\phi^{(n)}\right)-\left(\phi_{M}-\phi_{M}^{(n)}\right)=\left(\phi-\phi_{M}\right)+\left(\phi_{M}^{(n)}-\phi^{(n)}\right) \\
& \equiv \Delta_{M}+\Delta_{M}^{(n)}
\end{aligned}
$$

From (44) and inversions of (38) and (39),

$$
\text { (45) } \Delta_{M}=\mathcal{A}^{-1} f-\mathcal{B}^{-1} f=\mathcal{B}^{-1}(\mathcal{B}-\mathcal{A}) \mathcal{A}^{-1} f=\lambda \mathcal{B}^{-1}\left(\mathcal{K}-\mathcal{K}_{M}\right) \mathcal{A}^{-1} f .
$$

\footnotetext{
${ }^{3}$ Within the general framework of bounded projections on compact operators in Banach spaces, this result follows directly from lemma 3.1.2 which, of course, cannot reveal the above condition on differentiability off $K(x, s)$.
} 
Similarly, from (44) and inversions of (40) and (41),

$$
\Delta_{M}^{(n)}=-\lambda \mathcal{D}^{-1}\left(\mathcal{K}-\mathcal{K}_{M}\right) \mathcal{P}_{h}^{(n)} \mathcal{C}^{-1} f
$$

Alternatively from (44) and the existing forms of (38) and (39),

$$
\begin{aligned}
\Delta_{M} & =\lambda\left(\mathcal{K} \phi-\mathcal{K}_{M} \phi_{M}\right) \\
& =\lambda\left(\mathcal{K} \phi+\left(\mathcal{K}-\mathcal{K}_{M}\right) \phi_{M}-\mathcal{K} \phi_{M}\right) \\
& =\lambda\left(\mathcal{K} \Delta_{M}+\left(\mathcal{K}-\mathcal{K}_{M}\right) \phi_{M}\right) \\
\Rightarrow \quad \mathcal{A} \Delta_{M} & =\lambda\left(\mathcal{K}-\mathcal{K}_{M}\right) \phi_{M} \\
\Rightarrow \quad \Delta_{M} & =\lambda \mathcal{A}^{-1}\left(\mathcal{K}-\mathcal{K}_{M}\right) \mathcal{B}^{-1} f .
\end{aligned}
$$

Similarly, (44), (40) and (41) give

$$
\Delta_{M}^{(n)}=-\lambda \mathcal{C}^{-1}\left(\mathcal{K}-\mathcal{K}_{M}\right) \mathcal{P}_{h}^{(n)} \mathcal{D}^{-1} f .
$$

Using either form of $\Delta_{M}$ and $\Delta_{M}^{(n)}$, the error discrepancy is bounded according to

$$
\begin{aligned}
\left\|\Delta \mathcal{E}_{M}^{(n)}\right\| & \leq\left\|\Delta_{M}\right\|+\left\|\Delta_{M}^{(n)}\right\| \\
(49) \quad & \leq|\lambda|\left\|\mathcal{K}-\mathcal{K}_{M}\right\|\|f\|\left(\left\|\mathcal{A}^{-1}\right\|\left\|\mathcal{B}^{-1}\right\|+\left\|\mathcal{C}^{-1}\right\|\left\|\mathcal{D}^{-1}\right\|\left\|\mathcal{P}_{h}^{(n)}\right\|\right) \\
& \equiv \omega_{h}^{(n)}\left\|\mathcal{K}-\mathcal{K}_{M}\right\|,
\end{aligned}
$$

in which $\omega_{h}^{(n)}$ is bounded and independent of the differentiability of $\phi$ or its numerical/degenerate counterparts. Hence, by (43) and (49), the theoretically predicted error tends to the true numerical error as $M \rightarrow \infty$. Moreover, from (45) (or (47)) and (46) (or (48)), both $\Delta_{M}$ and $\Delta_{M}^{(n)}$ independently converge to zero at the same rate, $\left\|\mathcal{K}-\mathcal{K}_{M}\right\|$, irrespective of the degree of piecewise-polynomial interpolation or the numerical mesh size. How readily $K(x, s)$ admits a degenerate-kernel approximation is therefore the controlling factor in the rate at which the error discrepancy decreases. Note that we do not require $h \rightarrow 0$ in (49), the effect of which is to make each sub-term within the norm tend independently to zero. 
5. Implementation. The finite-rank degenerate-kernel approximation

$$
K_{M}(x, s)=\sum_{m=1}^{M} \theta_{m}(x) \psi_{m}(s)
$$

is constructed, in which the functions $\left\{\psi_{m}\right\}_{m=1}^{M}$ comprise an orthonormal polynomial basis $\mathcal{B}_{\psi}$ on $C[a, b]$ and the Fourier coefficients $\theta_{m}$ are given by

$$
\theta_{m}(x)=\int_{a}^{b} K(x, s) \psi_{m}(s) d s .
$$

A polynomial basis is used so that Weierstrass' approximation theorem may, for sufficiently large $M$, be applied with respect to the supremum norm on $C[a, b]$. Solution of (5) in the form

$$
\phi_{M}=f+\lambda \sum_{m=1}^{M} c_{m} \theta_{m} .
$$

using the finite-rank orthogonal expansion (50) proceeds along the lines of, e.g., $\S 2.4$ in Atkinson, (1997), the linear system $(\mathbf{I}-\lambda \mathbf{A}) \mathbf{C}=\mathbf{F}$ having $(\mathbf{A})_{i j}=\left\langle\psi_{i}, \theta_{j}\right\rangle$ and $(\mathbf{F})_{i}=\left\langle\psi_{i}, f\right\rangle$ for $i, j=1, \ldots, M$. All integrals in (51) and in the elements of $\mathbf{A}$ and $\mathbf{F}$ were evaluated using Gaussian quadrature, all calculations being performed in the $\mathrm{C}$ language on a standard laptop PC. Thus exact expressions for the integrations in (51) are not required, making the method widely applicable to all suitably behaved kernels $K(x, s)$ and bases $\mathcal{B}_{\psi}$. For the numerical experiments in $\S 6$ the orthonormal polynomial basis $\mathcal{B}_{\psi} \subseteq C[a, b]$ was constructed using a three-term recurrence relation as in Press et al. (1992, p.142) or Gautschi (2004, p.10). Here $\mathcal{B}_{\psi}$ has to be modified in a non-standard way (the details are omitted) because it can be shown that numerical instabilities swamp the calculations when $b-a>4$ and rounding errors pollute the calculations when $b-a<4$. Hence, irrespective of the interval $[a, b]$, all calculations in the generation of $\mathcal{B}_{\psi}$ and (51) were mapped onto the interval $[-2,2]$; symmetry about $x=0$ simplifies the computational construction of $\mathcal{B}_{\psi}$. The leadingorder (asymptotically consistent to order $\mathcal{O}\left(h^{\nu_{n}}\right)$ ) approximation of the numerical error incurred by projection of (2) - with non-degenerate 
kernel - onto a mesh of size $h$ is finally calculated using (29) and (37) in the form

$$
\mathcal{E}_{M}^{(n)}=\lambda\left((\mathbf{I}-\lambda \mathbf{A}+\lambda \delta \mathbf{A})^{-\mathbf{1}}\left(\delta \mathbf{F}+\lambda \delta \mathbf{A}(\mathbf{I}-\lambda \mathbf{A})^{-\mathbf{1}} \mathbf{F}\right)\right)^{\mathrm{T}} \boldsymbol{\theta},
$$

in which $(\mathbf{A})_{i j}=\left\langle\psi_{i}, \theta_{j}\right\rangle,(\mathbf{F})_{i}=\left\langle\psi_{i}, f\right\rangle,(\boldsymbol{\theta})_{i}=\theta_{i}$, and (13) and (17) give

$$
\left.\begin{array}{rl}
(\delta \mathbf{A})_{i j} & =\delta^{(n)}\left(\psi_{i}, \theta_{j}\right) \approx \chi_{0}^{(n)}\left(\psi_{i}, \theta_{j}\right) h^{\nu_{n}} \\
(\delta \mathbf{F})_{i} & =\delta^{(n)}\left(\psi_{i}, f\right) \approx \chi_{0}^{(n)}\left(\psi_{i}, f\right) h^{\nu_{n}}
\end{array}\right\}
$$

for $i, j=1, \ldots, M$ and $n=0,1,2$. In implementing (29), it is to be noted that the elements of the matrices and vectors in (52) are calculated using high-order Gaussian quadrature rules that are adapted interactively to guarantee an absolute error, in double-precision arithmetic, that is overwhelmingly dominated by the magnitude of $\mathcal{E}_{M}^{(n)}$ in any of our experiments. Hence our predictions based on (29) are not affected by using the approximation (52).

6. Results and discussion. We validate the result (52) on two test problems. The first example (Kress, 1989, Example 11.4),

$$
\phi(x)=e^{-x}+\frac{1}{2}\left(e^{-(x+1)}-1\right)+\frac{1}{2} \int_{0}^{1}(x+1) e^{-x s} \phi(s) d s,
$$

has a smooth kernel and solution $\phi(x)=e^{-x}$. The second example,

$$
\begin{aligned}
\phi(x)= & \frac{1}{2-x}+\arctan \left(\frac{4}{\sqrt{2+16 x^{2}}}\right) \\
& -\int_{-1}^{1} \frac{(2-s) \sqrt{2+16 x^{2}}}{1+8 x^{2}+8 s^{2}} \phi(s) d s
\end{aligned}
$$

has a sharply peaked kernel and solution $\phi(x)=(2-x)^{-1}$.

With $h=(b-a) / N$ and $n=0,1,2$, each projection has $N^{(n)}=$ $N+\frac{n}{2}(3-n)$ regularly spaced collocation nodes $x_{i}, i=1,2, \ldots, N^{(n)}$. 
For both examples (54) and (55), a value of $N=128$ was used in piecewise-constant $(n=0)$, linear $(n=1)$ and quadratic $(n=2)$ projections to obtain $\phi^{(n)}$ in the discrete problem (3). Since $\phi$ is known for these test problems, $\mathcal{E}^{(n)} \equiv \phi-\phi^{(n)}$ can be calculated directly and compared with our new estimate $\mathcal{E}_{M}^{(n)}$ in (52).

We first examine the invertibility condition (30) and the assertion that the bound (31) could be relaxed to (32) on the basis that, in practice, $\kappa \approx\|\delta \mathbf{X}\| \approx M h^{\nu_{n}}$. This assertion is clearly evidenced in figure 1, which shows that $\kappa / h^{\nu_{n}}$ increases linearly with $M$ for all $n$, with the welcome observation that the coefficient of proportionality is of order $\mathcal{O}(1)$ in both the smooth- and peaked-kernel problems. Hence (32) may be relaxed even further to

$$
M<\mathcal{O}\left(N^{\nu_{n}} / c_{n}\right), \quad c_{n}>\mathcal{O}(1),
$$

which all but guarantees the applicability of our method in practice. A notable feature of figure 1 (a) is that the error prediction when $n=1$ should be far superior to that when $n=0$, despite the fact that both methods are of the same order, $\mathcal{O}\left(h^{2}\right)$.
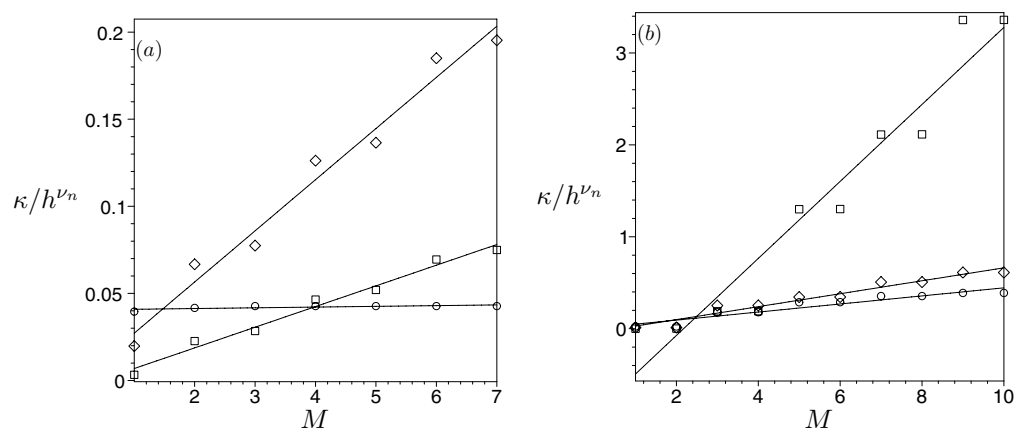

Figure 1. Linear variation of $\kappa / h^{\nu_{n}}$ with $M$ for (a) the smooth-kernel problem, (54), and (b) the peaked-kernel problem, (55), for $n=0(\diamond), n=1$ (०) and $n=2$ (ם). Note the different vertical scales in each figure. The straight lines are least-squares fits to the data generated by calculating $\kappa$ using (30) for increasingly large linear systems. Because of the order $\mathcal{O}(1)$ coefficients on the vertical axes, the invertibility criterion $\kappa<1$ is easily met even for moderate values of $N$. The greater gradient of the $n=2$ line in (b) is tempered by the fact that its data are coefficients of $h^{4} \ll 1$ in the bound (32). 
To quantify the error estimate globally on the interval $[a, b]$, we calculate the root-mean-square of the error discrepancy in (44) as

$$
\left\langle\Delta \mathcal{E}_{M}^{(n)}\right\rangle=\sqrt{h \sum_{i}\left(\Delta \mathcal{E}_{M}^{(n)}\right)_{i}^{2}},
$$

where the summation occurs over all collocation nodes $x_{i}$. Similarly, the root-mean-square numerical error is

$$
\left\langle\mathcal{E}^{(n)}\right\rangle=\sqrt{h \sum_{i}\left(\mathcal{E}^{(n)}\right)_{i}^{2}} .
$$

We calculate the global root-mean-square relative discrepancy using

$$
\rho_{M}^{(n)} \equiv \frac{\left\langle\Delta \mathcal{E}_{M}^{(n)}\right\rangle}{\left\langle\mathcal{E}^{(n)}\right\rangle}
$$

rather than via the local-based measure

$$
\sqrt{h \sum_{i}\left(1-\frac{\mathcal{E}_{M}^{(n)}}{\mathcal{E}^{(n)}}\right)_{i}^{2}},
$$

in anticipation of examples in which the exact and numerical solutions (almost) coincide at one or more collocation points.

For each polynomial interpolatory projection $(n=0,1,2)$ and example problem (54) or (55), figures 2,4 and 6 show plots of the error distributions $\mathcal{E}_{M}^{(n)}(x)$ throughout $[a, b]$, and figures figures 3,5 and 7 show the convergence (with $M$ ) of error discrepancies $\left\langle\Delta \mathcal{E}_{M}^{(n)}\right\rangle$. Also shown as a continuous line on the error-discrepancy plots 3,5 and 7 is the root-mean-square numerical error $\left\langle\mathcal{E}^{(n)}\right\rangle$ given by $(57)$.

The error-distribution plots 2,4 and 6 reveal the uniform convergence with $M$ throughout the interval $[a, b]$ of the theoretically predicted (continuous) error (52) to the computed numerical (discrete) error for $n=0,1,2$ with $N=128$. As expected, the convergence for the peaked-kernel problem (55) (figures (2) and (6)) is less rapid than for the smooth-kernel problem (54) (figure (4)). 
The error-discrepancy plots 3,5 and 7 clearly show that the initial convergence rate with $M$ is exponential, in each case leading to a relative-discrepancy (58) plateau of approximately $\mathcal{O}\left(10^{-5}\right)$. This initial exponential convergence towards the plateau occurs for all values of $n$ and $h$ (the same behavior occurs for other values of $N$ ), thereby corroborating the form of the error bound predicted in (49). Expressing the exponential convergence rates in the form $A e^{-B M}$, for the smoothkernel problem (54) we find through least-squares fitting (the dashed lines on figures 3,5 and 7 ) that $B \approx 2.735,3.882,2.806$ for $n=0,1,2$, showing that the actual rate of convergence does not vary dramatically with $n$. By contrast, for the peaked-kernel problem (55), we obtain $B \approx 0.316,1.231,0.313$ for $n=0,1,2$, so that our method is considerably faster when $n=1$ than it is when $n=0,2$; this discovery is quantified in $\S 7$. It is possible that this unexpected rapid convergence is a result of the coefficients $\chi_{0}^{(n)}$ in (17) enjoying their simplest form when $n=1$, a form that explicitly avoids differentiation of the orthonormal polynomial basis in the implementation.

Note that the values to which $\left\langle\Delta \mathcal{E}_{M}^{(n)}\right\rangle$ converge in each of figures 3,5 and 7 are not roundoff plateaux, but rather $\mathcal{O}\left(h^{\nu_{n}+2}\right)$ truncation plateaux resulting from the use of only the leading-order term in (13); these plateaux descend with increasing $N$.

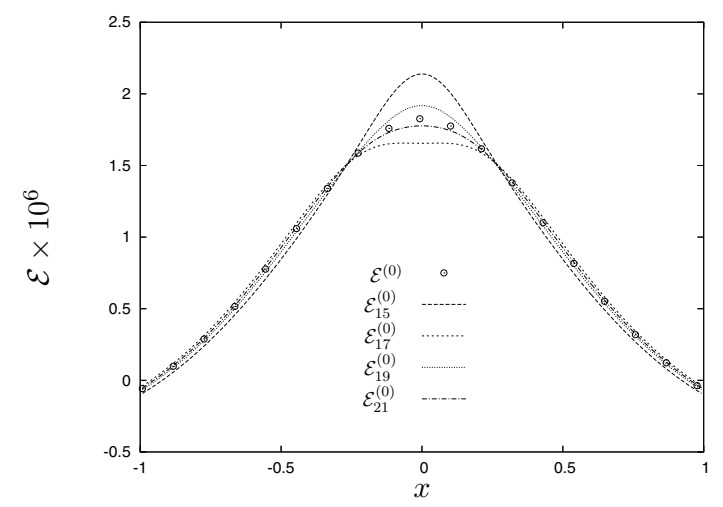

Figure 2. Plot showing the rapid convergence with $M$ of the piecewise-constant predicted error $\mathcal{E}_{M}^{(0)}$ (different lines for different $M$ ) to the actual piecewise-constant numerical error $\mathcal{E}^{(0)}$ (circles) for the solution of (55) with $N=128$. For clarity, $\mathcal{E}^{(0)}$ has been plotted at regularly spaced collocation points. 


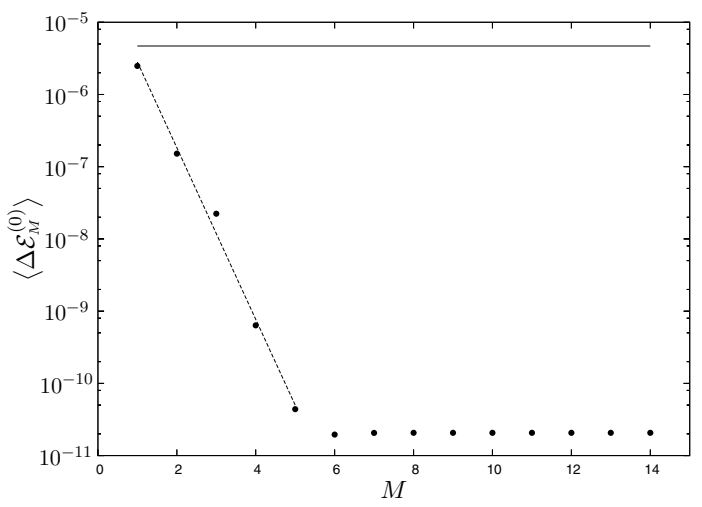

Figure 3. Logarithmic plot showing the rapid convergence with $M$ of the piecewiseconstant root-mean-square discrepancy $\left\langle\Delta \mathcal{E}_{M}^{(0)}\right\rangle$ of (56) between the predicted and actual numerical errors for the piecewise-constant projection method applied to (54) with $N=128$ nodes. The root-mean-square numerical error $\left\langle\mathcal{E}^{(0)}\right\rangle$ of $(57)$ over all nodes is shown by a continuous line. The dashed line is the least-squares fit $\Delta \mathcal{E} \approx 4.346 \times 10^{-5} \exp (-2.735 M)$ to the exponentially convergent data, which here converge to the $\mathcal{O}\left(h^{4}\right)$ truncation plateau, and not the roundoff plateau, since our analysis predicts the coefficient of the $\mathcal{O}\left(h^{2}\right)$ term in the numerical error.

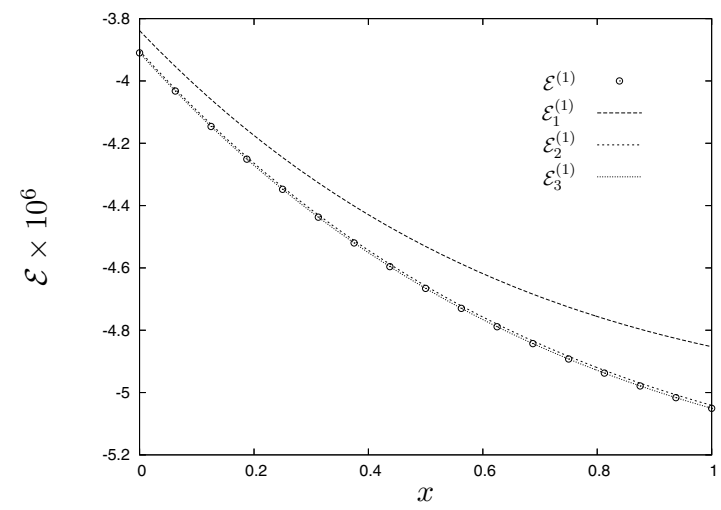

Figure 4. Plot showing the rapid convergence with $M$ of the piecewise-linear predicted error $\mathcal{E}_{M}^{(1)}$ (different lines for different $M$ ) to the actual piecewise-linear numerical error $\mathcal{E}^{(1)}$ (circles) for the solution of (54) with $N=128$. For clarity, $\mathcal{E}^{(1)}$ has been plotted at regularly spaced collocation points. 


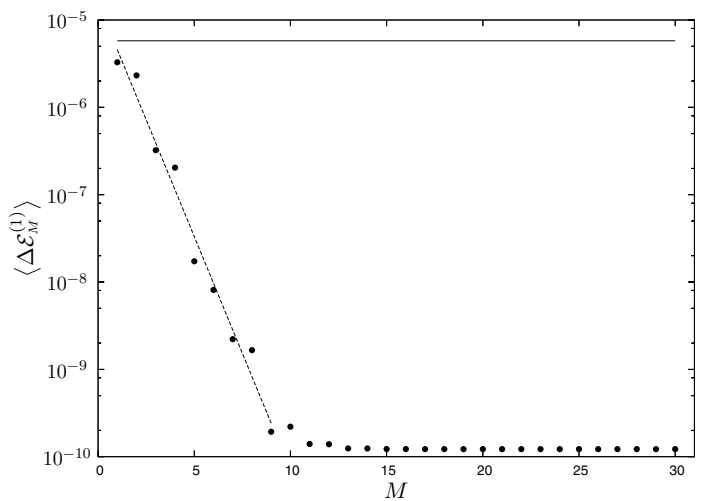

Figure 5. Logarithmic plot showing the rapid convergence with $M$ of the piecewiselinear root-mean-square discrepancy $\left\langle\Delta \mathcal{E}_{M}^{(1)}\right\rangle$ of (56) between the predicted and actual numerical errors for the piecewise-linear projection method applied to (55) with $N=128$ nodes. The root-mean-square numerical error $\left\langle\mathcal{E}^{(1)}\right\rangle$ of (57) over all nodes is shown by a continuous line. The dashed line is the least-squares fit $\Delta \mathcal{E} \approx 1.569 \times 10^{-5} \exp (-1.231 M)$ to the exponentially convergent data, which here converge to the $\mathcal{O}\left(h^{4}\right)$ truncation plateau, and not the roundoff plateau, since our analysis predicts the coefficient of the $\mathcal{O}\left(h^{2}\right)$ term in the numerical error.

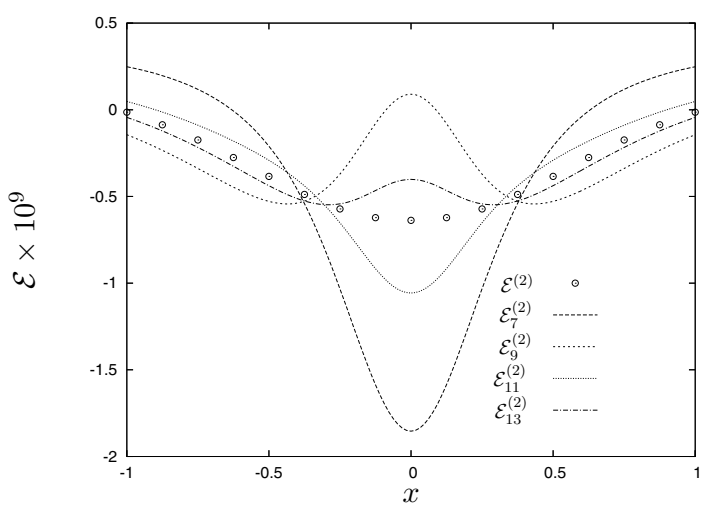

Figure 6. Plot showing the rapid convergence with $M$ of the predicted error $\mathcal{E}_{M}^{(2)}$ (different lines for different $M$ ) to the actual piecewise-quadratic numerical error $\mathcal{E}^{(2)}$ (circles) for the solution of $(55)$ with $N=128$. For clarity, $\mathcal{E}^{(2)}$ has been plotted at regularly spaced collocation points. Note the scaling on the vertical axis, showing $\mathcal{O}\left(h^{4}\right)$ behaviour in contrast to the $\mathcal{O}\left(h^{2}\right)$ behaviour in figures 2 and 4. 


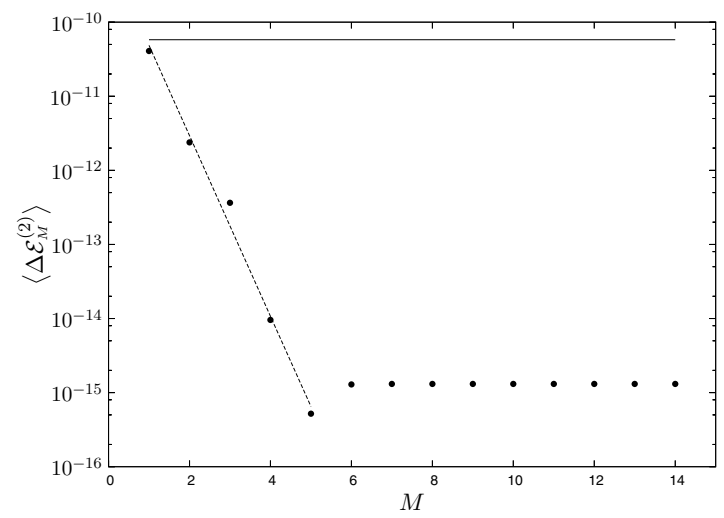

Figure 7. Logarithmic plot showing the rapid convergence with $M$ of the root-meansquare discrepancy $\left\langle\Delta \mathcal{E}_{M}^{(2)}\right\rangle$ of (56) between the predicted and actual numerical errors for the piecewise-quadratic projection method applied to (54) with $N=128$ nodes. The root-mean-square numerical error $\left\langle\mathcal{E}^{(2)}\right\rangle$ of $(57)$ over all nodes is shown by a continuous line. The dashed line is the least-squares fit $\Delta \mathcal{E} \approx$ $8.045 \times 10^{-10} \exp (-2.806 M)$ to the exponentially convergent data, which here converge to the $\mathcal{O}\left(h^{6}\right)$ truncation plateau, and not the roundoff plateau, since our analysis predicts the coefficient of the $\mathcal{O}\left(h^{4}\right)$ term in the numerical error.

Finally, we reconsider the effect of linearizing (29). The plots in figure 8 provide clear a posteriori evidence to support the use of the linearization (33), which is to be preferred since it requires that only one $M \times M$ linear system has to undergo a single LU decomposition and two backward and forward substitutions. Analogous to the behavior displayed by $\kappa / h^{\nu_{n}}$ in figure 1 , both $\left\|\delta \mathbf{C}^{(n)}\right\| / h^{\nu_{n}}$ and $\left\|\delta \mathbf{C}_{L}^{(n)}\right\| / h^{\nu_{n}}$ increase linearly with $M$. Note that the linear increase of $\left\|\delta \mathbf{C}^{(n)}\right\|$ with $M$ does not lead to unbounded estimates of $\left|\mathcal{E}_{M}^{(n)}\right|$ since, by writing (52) in the form

$$
\mathcal{E}_{M}^{(n)}(x) \approx \lambda \delta \mathbf{C}^{(n)} \cdot \boldsymbol{\theta}(x),
$$

it is clear that the exponentially decreasing magnitude of the norms of the components of the Fourier-coefficient vector $\boldsymbol{\theta}$ is the restraining factor. Specifically, for the smooth-kernel test problem (54), the $m$ th such component decays approximately according to the least-squares quadratic-exponential fit $\left\|\theta_{m}\right\| \approx e^{1.94-1.54 m-0.143 m^{2}}$ in which the small negative quadratic coefficient indicates super-exponential convergence for small $m$. For the peaked-kernel problem (55), the double 
pole in the kernel gives the two quadratic-exponential fits $\left\|\theta_{m}\right\|_{\text {odd }} \approx$ $e^{1.16-0.443 m+0.00361 m^{2}}$ and $\left\|\theta_{m}\right\|_{\text {even }} \approx e^{1.33-0.578 m+0.0111 m^{2}}$ in which the very small positive quadratic coefficients indicate marginally subexponential convergence for small $m$. Evident in figure 8 is the nowfamiliar observation that there is minimal linear growth when $n=1$; this, we suggest, is associated with the absence of differentiated polynomial basis functions in the expression for $\chi_{0}^{(1)}$ in (17).
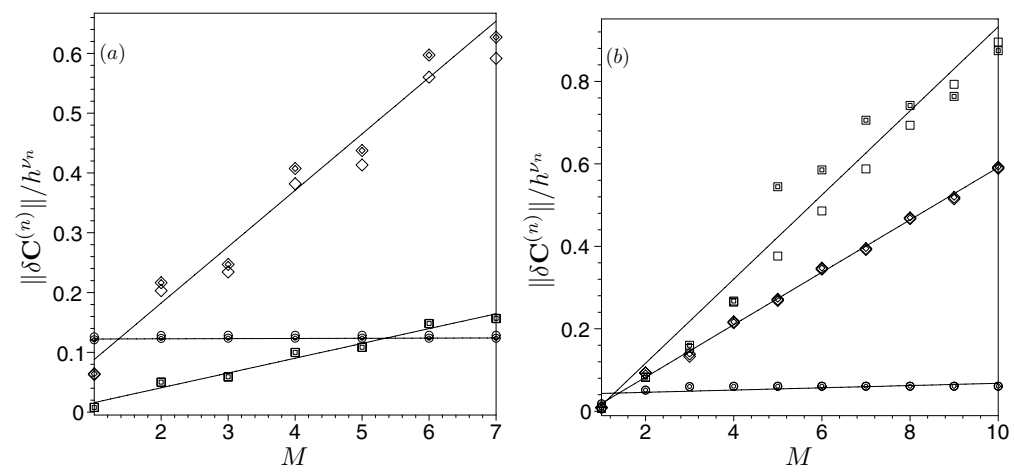

Figure 8. Linear variation of $\left\|\delta \mathbf{C}^{(n)}\right\| / h^{\nu_{n}}$ with $M$ for (a) the smooth-kernel problem, (54), and (b) the peaked-kernel problem, (55), for $n=0(\diamond), n=1$ (०) and $n=2(\square)$. For each $n$, the filled symbols are the values of $\delta \mathbf{C}^{(n)}$ from the non-linear (29) and the hollow symbols are the values of $\left\|\delta \mathbf{C}_{L}^{(n)}\right\| / h^{\nu_{n}}$ computed using the linearization (33). The straight lines are least-squares fits to the filled symbols. The agreement between the non-linear and linearized theory provides $a$ posteriori justification for the latter, even in the peaked-kernel case. The negligible gradient of the $n=1$ line in (b) accounts for the rapid descent to the truncation plateau in figure 5 in the peaked-kernel case. The greatest discrepancy between linear and non-linear results, for $n=2$ in (b), is tempered by the fact that the data are coefficients of $h^{4} \ll 1$. Note the similar vertical scales in each figure.

Table 1 on the next page provides further evidence to justify the linearisation by showing the actual the error discrepancy $\Delta \mathcal{E}_{M}^{(n)}$ predicted by using $\delta \mathbf{C}^{(n)}$ and $\delta \mathbf{C}_{L}^{(n)}$ in (37). All calculations were undertaken in the $\mathrm{C}$ language on an $866 \mathrm{Mhz}$ Pentium $3 \mathrm{PC}$ with 512Mb RAM.

7. Efficiency relative to extrapolation. The complexity of the new algorithm precludes a meaningful explicit analysis of its cost rel- 


\begin{tabular}{|c||c|c|c|}
\hline \multicolumn{4}{|c|}{$\Delta \mathcal{E}_{M}^{(n)}$ for smooth-kernel problem (54) with $M=10$ and $N=128$} \\
\hline \hline & $n=0$ & $n=1$ & $n=2$ \\
\hline \hline$\delta \mathbf{C}^{(n)}$ in (37) & $2.066 \mathrm{e}-11$ & $1.617 \mathrm{e}-11$ & $1.310 \mathrm{e}-15$ \\
\hline$\delta \mathbf{C}_{L}^{(n)}$ in (37) & $8.912 \mathrm{e}-12$ & $2.728 \mathrm{e}-11$ & $1.320 \mathrm{e}-15$ \\
\hline
\end{tabular}

\begin{tabular}{|c||c|c|c|}
\hline \multicolumn{4}{|c|}{$\Delta \mathcal{E}_{M}^{(n)}$ for peaked-kernel problem (55) with $M=30$ and $N=128$} \\
\hline \hline & $n=0$ & $n=1$ & $n=2$ \\
\hline$\delta \mathbf{C}^{(n)}$ in (37) & $1.103 \mathrm{e}-09$ & $1.221 \mathrm{e}-10$ & $5.603 \mathrm{e}-13$ \\
\hline$\delta \mathbf{C}_{L}^{(n)}$ in (37) & $8.430 \mathrm{e}-10$ & $1.436 \mathrm{e}-10$ & $5.603 \mathrm{e}-13$ \\
\hline
\end{tabular}

Table 1. Comparison of error discrepancies $\Delta \mathcal{E}_{M}^{(n)}$ obtained using $\delta \mathbf{C}^{(n)}$ and its linearized counterpart $\delta \mathbf{C}_{L}^{(n)}$. Note the minimized relative differences when $n=2$, in keeping with expressions (35) and (36), only the latter of which has a linear error form at order $\mathcal{O}\left(h^{\nu_{n}+2}\right)$. Computation times for each of the smooth-kernel results were 0.199 and 0.013 seconds when using 96 and 10 Gauss points respectively for evaluating the system-matrix elements.

ative to Richardson extrapolation. Instead, we examine the efficiency ratio $R_{h}^{(n)}$ of actual CPU times on the same computational platform required by both the present method and Richardson extrapolation to obtain relative error discrepancies (58) comparable to those at the truncation plateau. Based upon the evidence in figure 8 and table 1 , we use the linearized inversion (33). We assume that $\nu_{n}$ is known to both methods so that Richardson extrapolation requires only two, rather than three, independent numerical solutions. Efficiency ratios for $n=0,1,2$ and $N=64,128,256$ are given in tables 2 and 3 , in each of which an efficiency ratio greater than unity indicates the factor by which the new method is faster than Richardson extrapolation, which is here based on solutions with $N$ and $2 N$ intervals on $[a, b]$.

Table 2 provides convincing evidence for the motivation of the new method. For the smooth-kernel problem, the efficiency factors are impressive for low values of $N$, and improve dramatically with increasing $N$. As expected, the efficiency factors decrease in the case of the peaked kernel, as demonstrated in table 3. Of note, however, is the manifestation of our discovery in $\S 6$ regarding the case $n=1$ when, even for low $N$, the new method is considerably more efficient than Richardson ex- 
trapolation; as $N$ increases, the advantage of the new method spreads to all values of $n$. When the full non-linear inversion (29) was used, the efficiency ratios in tables 2 and 3 decreased by an average (over each set of nine values) of only $0.515 \%$ and $0.255 \%$ respectively.

\begin{tabular}{|c||r|r|r|}
\hline \multicolumn{4}{|c|}{$R_{h}^{(n)}$ for smooth-kernel problem (54) } \\
\hline \hline & $n=0$ & $n=1$ & $n=2$ \\
\hline \hline$N=64$ & 232.79 & 390.76 & 208.33 \\
\hline$N=128$ & 2195.21 & 1052.32 & 632.33 \\
\hline$N=256$ & 13607.98 & 3141.16 & 2104.14 \\
\hline
\end{tabular}

Table 2. Speed-up factors of the linearized method relative to Richardson extrapolation for the iterated collocation solution of the smooth-kernel problem (54). Results from the new method for $n=0,1,2$ were obtained using $M=6,4,5$ respectively. Global integrals were evaluated using 10-point Gaussian quadrature.

\begin{tabular}{|c||r|r|r|}
\hline \multicolumn{3}{|c|}{$R_{h}^{(n)}$ for peaked-kernel problem (55) } \\
\hline \hline & $n=0$ & $n=1$ & $n=2$ \\
\hline \hline$N=64$ & 3.65 & 77.16 & 1.06 \\
\hline$N=128$ & 48.64 & 210.35 & 4.75 \\
\hline$N=256$ & 362.59 & 389.69 & 23.16 \\
\hline
\end{tabular}

Table 3. Speed-up factors of the linearized method relative to Richardson extrapolation for the iterated collocation solution of the peaked-kernel problem (55). Results from the new method for $n=0,1,2$ were obtained using $M=34,11,34$ respectively. For $n=0,2$, the truncation plateaux were not reached and the comparison was based upon a relative error discrepancy of order $\mathcal{O}\left(10^{-4}\right)$. Global integrals were evaluated using 64-point Gaussian quadrature.

We also note that the new method provides the error discrepancy $\mathcal{E}_{M}^{(n)}$ as an explicit function of $x$ over $[a, b]$ whereas $\mathcal{E}^{(n)}$ is known at only the collocation nodes. Moreover, in the context of practical applications, given an error tolerance $\epsilon>0$, the new method is useful for obtaining cheap a priori estimates of the value of $N$ that would guarantee $\mathcal{E}^{(n)} \leq \epsilon$. 
Finally, we remark that the present method does not accurately predict the errors incurred in integral equations for which the kernel is highly oscillatory on $[a, b]$ : it obtains the correct order, but not the right distribution with $x \in[a, b]$. Via (49), this is due to the fact that $\left\|\mathcal{K}-\mathcal{K}_{M}\right\|$ exhibits slow convergence with $M$ when $K$ is oscillatory.

Acknowledgments. The authors would like to thank Professor Kendall E Atkinson for helpful and encouraging detailed comments on previous drafts of this manuscript. The stimulating and constructive suggestions of an anonymous referee are also gratefully acknowledged.

\section{REFERENCES}

1. K. E. Atkinson, The Numerical Solution of Integral Equations of the Second Kind, Cambridge University Press (1997).

2. C. T. H. Baker, The Numerical Treatment of Integral Equations, Clarendon Press (1977).

3. D. R. Dellwo, Accelerated degenerate-kernel methods for linear integral equations., J. Comp. Appl. Math.,(1995) 58:135-149.

4. W. Gautschi, Orthogonal Polynomials: Computation and Approximation, Oxford University Press (2004).

5. G. Golub and V. Loan, Matrix Computations, John Hopkins (1989).

6. W. Hackbusch, Integral Equations: Theory and Numerical Treatment, Birkhäuser (1995).

7. S. Heinrich, On the optimal error of degenerate-kernel methods., J. Integral Equations, (1985) 9:251-266.

8. F. B. Hildebrand, Methods of Applied Mathematics, Prentice-Hall (1952).

9. R. Kress, Linear Integral Equations, Springer-Verlag. Applied Mathematical Sciences, Vol. 82 (1989).

10. P. Linz, Bounds and estimates for condition numbers of integral equations, SIAM J. Numer. Anal., (1991) 28:227-235.

11. W. H. Press, S.A. Teukolsky, W. T. Vetterling, and B. P. Flannery, Numerical Recipes in Fortran, Cambridge University Press (1992).

12. I. H. Sloan, Convergence of degenerate-kernel methods., J. Austral. Math. Soc. (series B), (1976a) 19:422-431.

13. I. H. Sloan, Error analysis for a class of degenerate-kernel methods, Numer. Math., (1976b) 25:231- 238.

14. I. H. Sloan, B. Burn and N. Datyner, A new approach to the numerical solution of integral equations, J. Comput. Phys., (1975) 18:92-105. 
15. R. Whitley, The stability of finite-rank methods with applications to integral equations, SIAM J. Numer. Anal., (1986) 23:118-134.

Department of Applied Mathematics, University of Leeds, Leeds LS2 9JT, ENGLAND

Email address: mark@maths.leeds.ac.uk 